\title{
OBSERVATIONS OF V592 CAS-AN OUTFLOW AT OPTICAL WAVELENGTHS
}

\author{
S. KAFKA ${ }^{1}$, D. W. Hoard ${ }^{1}$, R. K. Honeycutt ${ }^{2}$, and C. P. Deliyannis ${ }^{2}$ \\ ${ }^{1}$ Spitzer Science Center/Caltech, 220-6, 1200 E. California Blvd, Pasadena, CA 91125, USA; stella@ipac.caltech.edu \\ ${ }^{2}$ Indiana University, Astronomy Department, Swain Hall West, Bloomington, IN 47405, USA \\ Received 2008 May 27; accepted 2008 October 7; published 2008 December 10
}

\begin{abstract}
We present new red optical spectra of V592 Cas aimed at exploring the properties of the outflow of this system in a spectral region where the underlying white dwarf and the accretion disk do not contribute significantly to the observed absorption components of the $\mathrm{H} \alpha$ and $\mathrm{He}$ I line profiles. We use the $\mathrm{H} \alpha$ emission line to study the wind, which appears as pronounced blueshifted P Cygni absorption troughs whose low velocity end contaminates the blue side of the emission line profile. The wind appears to be episodic in nature, with multiple events reaching velocities of $5000 \mathrm{~km} \mathrm{~s}^{-1}$ in $\mathrm{H} \alpha$. Similar (but weaker) wind signatures appear in the He $\mathrm{I}$ $5876 \AA$ line but are absent in He I $6678 \AA$. Our data suggest that during wind episodes the wind is phase dependent and is visible for half of the orbit of the system. Considering that V592 Cas is viewed almost faceon, the symmetry axis of the outflow cannot be orthogonal to the disk and/or the outflow must have some other inherent asymmetry in outflow geometry. A possible origin of the wind is in a disk hotspot, either at the initial impact point of the accretion stream on the disk edge or as a result of disk overflow (similar to SW Sextantis stars). Simultaneous optical photometry during one night of our spectroscopic observations indicate that there is no clear relationship between the optical brightness variations and the strength of the outflow in this system.
\end{abstract}

Key words: novae, cataclysmic variables - stars: individual (V592 Cas) - stars: mass loss - stars: winds, outflows

\section{INTRODUCTION}

Cataclysmic variables (CVs) are semidetached binary stars in which a white dwarf (WD) is accreting H- or He-rich material from its low mass, usually main sequence, companion. In most cases, matter is transferred onto the WD via an accretion disk (disk CVs) whereas when the magnetic field of the WD is strong $\left(B \geqslant 10^{7} \mathrm{G}\right)$ an accretion column leads material directly onto the magnetic pole(s) of the WD via its magnetic field lines (magnetic CVs).

The evolution of CVs and all interacting binary stars (and hence knowledge of their progenitors and descendants) is driven by transfer and loss of mass and angular momentum. According to the standard scenario for $\mathrm{CV}$ evolution, angular momentum is lost from the system via magnetic braking and/or gravitational radiation (Spruit \& Ritter 1983). Existing models suggest that magnetic braking alone may not provide enough angular momentum loss to account for high mass transfer rate $(M) C V s$ above the gap (Taam \& Spruit 2001). An alternative mechanism for $\mathrm{CV}$ angular momentum loss could be provided by gravitational torques from a circumbinary (CB) disk (Taam \& Spruit 2001). This mechanism is provided via a disk wind resulting from the accretion process, whose strength depends explicitly on mass transfer (consequential mass loss) and may contribute significantly to the evolution of the system (Cannizzo \& Pudritz 1988).

Outflows in CVs have been observed in the UV by the International Ultraviolet Explorer (IUE) satellite and the Space Telescope Imaging Spectrograph (STIS) on the Hubble Space Telescope (HST), as P Cygni profiles in species such as C IV (1548 ̊ \& $1551 \AA)$, N v (1239 ̊ \& $1243 \AA$ ), and Si IV (1397 $)$, with maximum observed blueshifted absorption reaching $5000 \mathrm{~km} \mathrm{~s}^{-1}$ (e.g., Prinja et al. 2004) indicating wind mass loss rates as high as a percentage of the mass transfer rate $\left(\dot{M} \sim 5 \times 10^{-10} M_{\text {sun }} \mathrm{yr}^{-1}\right.$; e.g., Proga 1999). Until recently, only BZ Cam has been known to have reliable P Cygni profiles at optical wavelengths (Ringwald \& Naylor 1998) as well as in the far-UV (Hollis et al. 1992). However, BZ Cam is an unusual system in many respects (e.g., it is the only CV embedded in a nebula that is not associated with a recorded nova explosion) and may not be representative of disk CVs. Recent investigations have demonstrated that outflows in CVs can be observed in the optical regime using appropriate wind diagnostics. In a spectroscopic study of the old nova, Q Cyg, Kafka et al. (2003) examined time-resolved optical spectra in which pronounced P Cygni profiles were present in the He I triplet lines at $5876 \AA$ and $7065 \AA$ and in $\mathrm{H} \alpha$, indicating the presence of an outflow with velocity reaching $1500 \mathrm{~km} \mathrm{~s}^{-1}$. Using those lines as wind diagnostics, more disk $\mathrm{CVs}$ were discovered to display wind signatures in their optical spectra (Kafka \& Honeycutt 2004). The use of the He I triplets as outflow tracers provides a new tool for studying $\mathrm{CV}$ disk winds and their characteristics using ground-based facilities. Therefore, we initiated a survey of outflows from high mass transfer rate disk CVs, looking for wind signatures in the $\mathrm{He}$ I triplets and/or in the $\mathrm{H} \alpha$ line. The primary goal of the survey is to determine if $\mathrm{CV}$ winds can provide an effective mechanism of angular momentum loss that contributes to the secular evolution of those systems.

One target of our survey is V592 Cas, a disk CV discovered by Greenstein et al. (1970) via its very blue color and spectral characteristics. Spectroscopic observations of this system were among a collection of previously "poorly studied" CVs presented in Downes et al. (1995). The spectrum displays strong He II $4686 \AA$ and Bowen blend (C III/N III) emission and weak Balmer and He I emission lines. The first time-resolved optical spectroscopic study of the system (Huber et al. 1998) derived an initial orbital period of $2.472 \mathrm{hr}$. This was later revised by Taylor et al. (1998; hereafter T98) and Witherick et al. (2003; hereafter W03) to $P_{\text {orb }}=2.76 \mathrm{hr}$, placing the system toward the long period end of the CV period gap. Although it is not eclipsing ( $i \sim 28^{\circ}$; Huber et al. 1998) interest in V592 Cas 
Table 1

Log of Observations of V592 Cas

\begin{tabular}{|c|c|c|c|c|}
\hline UT Date & $\begin{array}{l}\text { Telescope/ } \\
\text { Instrument }\end{array}$ & & $\begin{array}{c}\text { No. } \\
\text { Exposures }\end{array}$ & $\begin{array}{c}\text { Integration Time } \\
\text { (s) }\end{array}$ \\
\hline Spectroscopy & & Wavelength Coverage & & \\
\hline 2005 Sep 9 & WIYN 3.5 m/Hydra MOS & $5500-8000 \AA$ & 14 & 600 \\
\hline \multirow[t]{2}{*}{2005 Oct 8} & KPNO 2.1 m/GoldCam & $5500-8000 \AA$ & 2 & 180 \\
\hline & & $5500-8000 \AA$ & 25 & 300 \\
\hline 2005 Oct 11 & & $5500-8000 \AA$ & 30 & 180 \\
\hline Photometry & & Filter & & \\
\hline 2005 Oct 8 & WIYN $0.9 \mathrm{~m} / \mathrm{S} 2 \mathrm{~KB}$ & $V$ & 58 & 30 \\
\hline
\end{tabular}

increased due to its diverse periodicities; it has both positive and negative superhumps (with periods of $P_{\text {orb }}+0.062$ days and $P_{\text {orb }}-0.028$ days; T98) as well as a suggested 21 minutes photometric oscillation (Kato \& Starkey 2002). In the UV, the system displays a rich suite of emission lines ( $\mathrm{C}$ III, $\mathrm{P}_{\mathrm{V}}, \mathrm{S}$ IV, $\mathrm{O}$ VI, and $\mathrm{Ly} \beta$ ) most of which are affected by strong and variable P Cygni profiles, indicating the presence of a wind reaching velocities of 2500-3000 $\mathrm{km} \mathrm{s}^{-1}$ (Prinja et al. 2004). An outflow was also found to be present in the optical spectra of the system (T98; W03).

In this paper, we present new multiepoch time-resolved red spectroscopy of V592 Cas in which the underlying absorption features from the WD and the accretion disk are not present. The purpose of our study is to characterize the outflow of the system as it appears in the $\mathrm{H} \alpha$ line and the He I triplets, and examine correlations with variations in the optical light curve. Our observations and data reduction techniques are described in Section 2, and our analysis of the data follows in Section 3. We finish by reviewing our main conclusions of this work in Section 4.

\section{OBSERVATIONS}

\subsection{Optical Spectroscopy}

Our spectroscopic data were obtained using the WIYN ${ }^{3}$ $3.5 \mathrm{~m}$ telescope/Hydra multiobject spectrograph (MOS) and the KPNO $2.1 \mathrm{~m}$ telescope/GoldCam spectrometer during four nights in 2005 September-October. For the Hydra MOS data, the 600 line $\mathrm{mm}^{-1}$ grating was used in first order, blazed at $7500 \AA$; the spectral coverage was $5500-8000 \AA$, with a resolution of $\sim 3 \AA$. The weather was fair, therefore our exposure times were fixed at $600 \mathrm{~s}$. For sky subtraction we used information from numerous unparked fibers; a CuAr lamp was used for wavelength calibration. For the GoldCam observations, we used grating 35 with a spectral coverage of 5300-8600 $\AA$ and a resolution of $\sim 3 \AA$. Exposure times ranged between 180 and $300 \mathrm{~s}$; the shorter exposure times were used in order to resolve rapid changes in the $\mathrm{H} \alpha$ profile when the weather permitted. A HeNeAr lamp was employed for wavelength calibration.

Dome flat fields were obtained for all data sets; flat-field corrections, as well as bias and sky subtraction, were performed using the standard IRAF $^{4}$ procedures. For the GoldCam data the IRAF packages apextract and apall were used for extracting

\footnotetext{
3 The WIYN Observatory is a joint facility of the University of Wisconsin-Madison, Indiana University, Yale University, and the National Optical Astronomy Observatory.

4 IRAF is distributed by the National Optical Astronomy Observatory, which is operated by the Association of Universities for Research in Astronomy, Inc. under cooperative agreement with the National Science Foundation.
}

one-dimensional spectra. Reductions of the Hydra MOS data used the IRAF twodspec and onedspec packages. The reduction steps included extraction of one-dimensional star and sky fiber spectra, flat-field correction, wavelength calibration, correction for scattered light, and sky subtraction. No corrections were made for strong telluric absorption features such as the $A$ and $B$ bands of $\mathrm{O}_{2}$. However, the relatively weak telluric $\mathrm{H}_{2} \mathrm{O}$ absorption in the region 5900-6020 $\AA$ was removed because of its occasional effect on the profile of $\mathrm{H} \alpha$. We interactively corrected the optical spectra using data from the Wallace \& Livingston (2003) atlas and the IRAF task telluric; a demonstration of the procedure is presented in Kafka et al. (2008) and is not repeated here.

\subsection{Optical Photometry}

Simultaneous optical photometry was obtained during the night of 2005 October 08 UT using a Johnson $V$ filter on the S2KB CCD camera on the WIYN $0.91 \mathrm{~m}$ telescope located at Kitt Peak, Arizona. We used $30 \mathrm{~s}$ for all of our exposures. Bias subtraction and dome flat fielding were applied using standard IRAF routines followed by APPhot aperture photometry. Incomplete ensemble photometry (Honeycutt 1992) was then used to reduce the data. The instrumental magnitudes from the WIYN $0.91 \mathrm{~m} \mathrm{~V}$-band photometry were placed on a standard magnitude scale using secondary standards No. 4 and No. 14 from Henden \& Honeycutt (1995). Information on this photometry is also included in Table 1.

\section{ANALYSIS}

Figure 1 presents the average red spectrum of V592 Cas, with the main absorption and emission features labeled; the spectrum is continuum normalized. The equivalent widths (EWs) and full width at half-maximum (FWHM) of the Gaussian fits to the major emission features in our spectra are presented in Table 2.

V592 Cas resembles a typical low inclination disk-accreting nova-like $\mathrm{CV}$, exhibiting a single-peaked $\mathrm{H} \alpha$ emission line and He I $5876 \AA, 6678 \AA$, and $7065 \AA$ emission. Noteworthy is the likely detection of C IV $(5801,5812 \AA)$ emission in our average spectrum. The presence of this line was also reported in T98 and it could indicate the presence of a strong ionization field in the system $^{5}$ or a C-rich donor star. In our data the detection appears marginal and overlaps with a diffuse interstellar absorption band (DIB) in the blue end and with the wings of the He I

\footnotetext{
5 This $\mathrm{C}$ IV line likely originates from a $\mathrm{C} \mathrm{V} \rightarrow \mathrm{C}$ IV recombination cascade requiring $490 \mathrm{eV}$.
} 


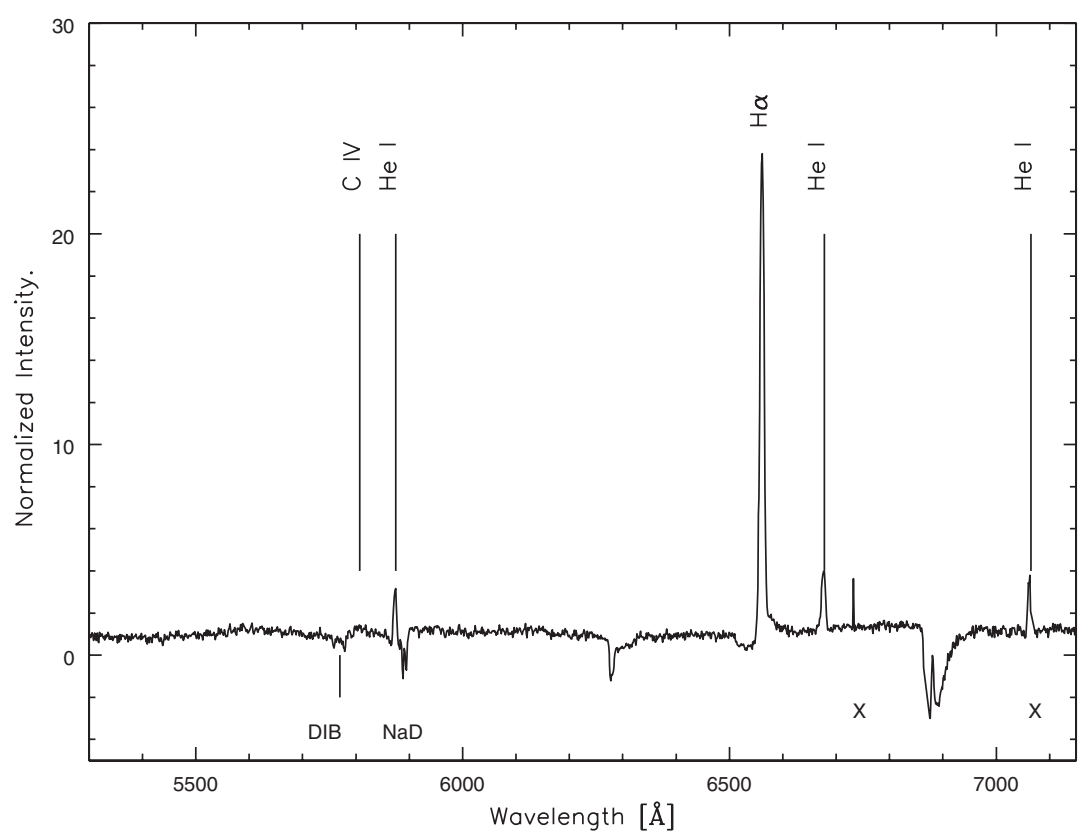

Figure 1. Average red spectrum of V592 Cas with emission/absorption features marked. Defects (blocked columns) in the spectrum that were corrected for presentation purposes are marked with an "X." The spectrum is continuum normalized.

Table 2

Measurements of the Emission Lines in the Average Spectrum of V592 Cas

\begin{tabular}{lccc}
\hline \hline Line & $\begin{array}{c}\text { Rest Wavelength } \\
(\AA)\end{array}$ & GFWHM $^{\mathrm{a}}$ & $\begin{array}{c}\mathrm{EW}^{\mathrm{b}} \\
(\AA)\end{array}$ \\
\hline $\mathrm{He} \mathrm{I}$ & 5876 & 5.223 & -0.354 \\
$\mathrm{H} \alpha$ & 6563 & 8.790 & -6.078 \\
$\mathrm{He} \mathrm{I}$ & 6675 & 8.155 & -0.722 \\
$\mathrm{He} \mathrm{I}$ & 7065 & 6.926 & -0.480 \\
\hline
\end{tabular}

Notes.

a GFWHM: full width at half-maximum of the Gaussian fit.

b We follow the IRAF convention, where negative $\mathrm{EW}$ values correspond to the strength of emission lines.

wind in the red, therefore it is not possible to measure the EW of the line. Also, we do not see any spectral features of the donor star, even when we combine all our individual spectra to maximize the signal-to-noise ratio $(\mathrm{S} / \mathrm{N})$. A striking feature of this red spectrum of V592 Cas is the presence of a deep blueshifted absorption trough (P Cygni profile) accompanying the $\mathrm{H} \alpha$ emission line; the sharp drop in the blue wing of the emission attests to the wind contaminating the emission line profile at low velocities. The absence of WD accretion disk absorption contaminating this line makes it an ideal tool for the study of the outflow in V592 Cas. The P Cygni profiles in the He I $5876 \AA$ line also help to determine the characteristics of the wind; however, the absorption trough in this line is much weaker than in $\mathrm{H} \alpha$. Therefore, $\mathrm{H} \alpha$ will be the primary tool for our analysis of the wind characteristics.

Using the IRAF " $\mathrm{k}$ " routine in the splot task and a single Gaussian fit, we measured the radial velocities (RVs) and EWs of the emission lines of $\mathrm{He} \mathrm{I}$ and $\mathrm{H} \alpha$. By an unfortunate coincidence, a blocked column on the GoldCam CCD (KPNO $2.1 \mathrm{~m}$ ) intercepted the He I $7065 \AA$ line. In order to include this line in our analysis we corrected the defect by visual interpolation using IRAF's splot; nonetheless determination of
EW and RVs from this line have larger errors and will not be weighted heavily in our conclusions. To exclude possible RV variations resulting from the wavelength calibration process of our data (due to an instrumental drift on the spectrograph) we measured the RVs of the telluric $\mathrm{O}_{2}(6280 \AA$ and $6867.2 \AA)$ bands in the spectra and examined for any variations in the wavelength, finding none of significance.

W03 derived an orbital period for the system based on the $\mathrm{H} \beta, \mathrm{H} \gamma$, and $\mathrm{He}$ II $4686 \AA$ emission lines. This He II line can have a wind origin or be strongly affected by it (Honeycutt et al. 1986; Marsh \& Horne 1990). T98 notice that the Balmer lines are highly variable in their spectra on different nights of their observations due to the presence of a wind, therefore they were not suited for a period study of the system; they used the He I $5876 \AA$ and $6678 \AA$ emission lines instead. We have used the spectroscopic ephemeris of T98 for our analysis. Efforts to improve the ephemeris of V592 Cas were thwarted by the relatively long intervals between epochs in the literature, leading to cycle count ambiguities when using the period errors provided in the literature. Also, the relatively short (four weeks) interval of our data made it impossible to improve the period using our data alone. We can however provide an updated JD0 = $2,453,654.88 \pm 0.02$ valid for the epoch of our data. This is for the - to + crossing of $\gamma$ for the He I lines, and was calculated by noting that this crossing occurs at phase $0.19 \pm 0.02$ (see Figure 2 and Table 3) for our data, using the T98 ephemeris. Because our interest is more in the character of the phase dependences than in the absolute phases, the remaining uncertainties in the ephermeris do not affect our analysis.

The resulting plots for our RVs of He I $5876 \AA$ A, He I $6678 \AA$, and $\mathrm{H} \alpha$ emission lines appear in Figures 2 and 3 (the $\mathrm{He}_{\mathrm{I}}$ 7065 A line exhibits a similar behavior to the other He I lines, albeit with more scatter). All He I lines appear to be sinusoidally modulated with the orbital phase. Using sinusoids of the form $v(t)=\gamma+K \sin \left[2 \pi\left(\phi-\phi_{0}\right)\right]$ in individual RV curves from each night we derived the systemic velocity $(\gamma)$, the semiamplitude of the sinusoidal fit $(K)$, and the gamma-crossing phase $\left(\phi_{0}\right)$ values listed in Table 3. Note the changes in $\gamma$ of the He I emission lines 

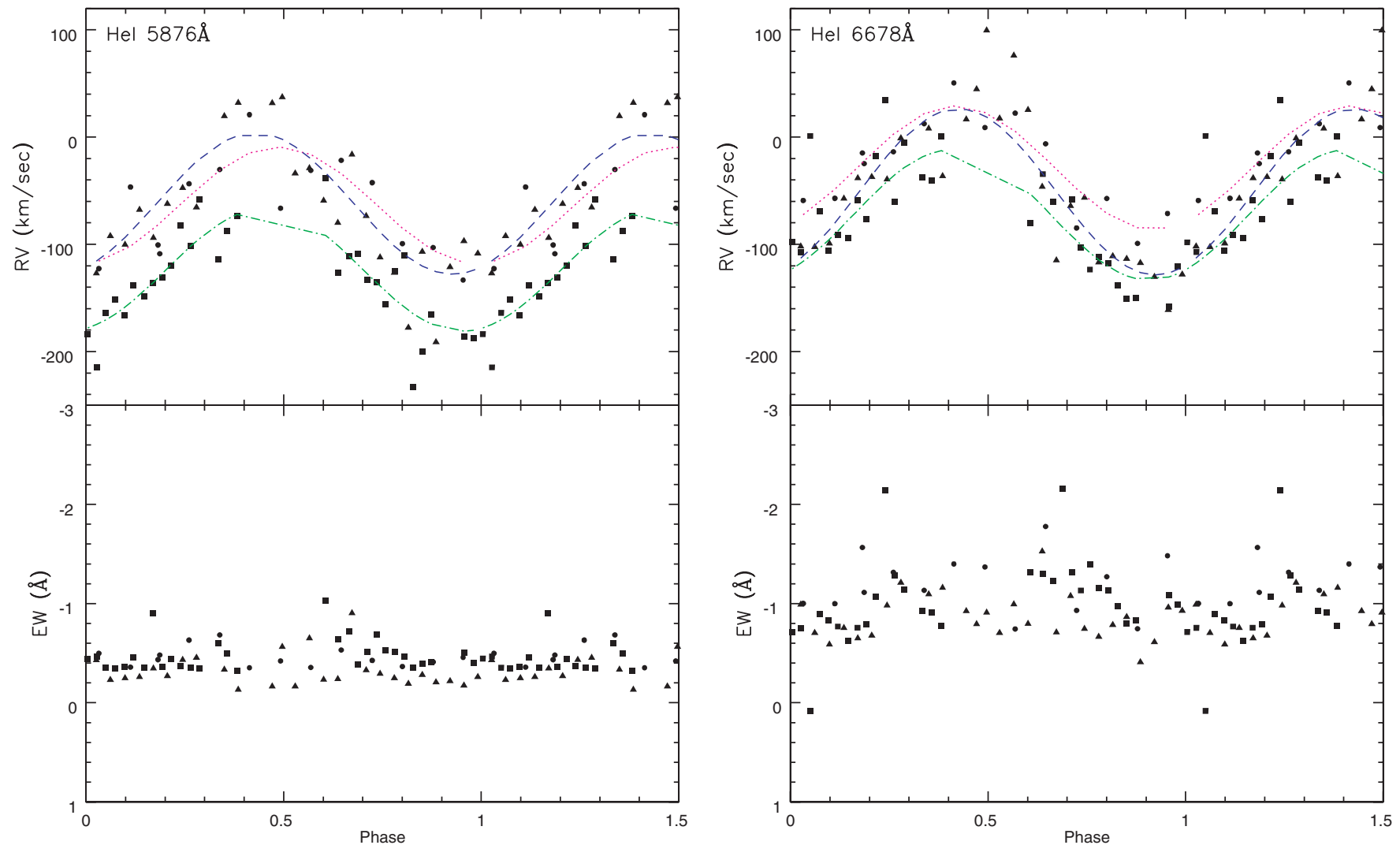

Figure 2. RV (top) and EW (bottom) of He I $5876 \AA$ and $6675 \AA$ emission lines. The triangles are used for the 2005 September 9 data, the squares for the 2005 October 8 data, and the circles for the 2005 October 11 data. The parameters of the RV fits at each epoch of our observations are presented in Table 3: the magenta dotted line is for the 2005 September 9 data, the blue dashed line is for the 2005 October 8 data, and the green dot-dashed line is for the 2005 October 11 data. Note that the asymmetry in the 2005 October 11 line is due to lack of data between phases 0.4 and 0.6 on that night of observations. See the text for details.

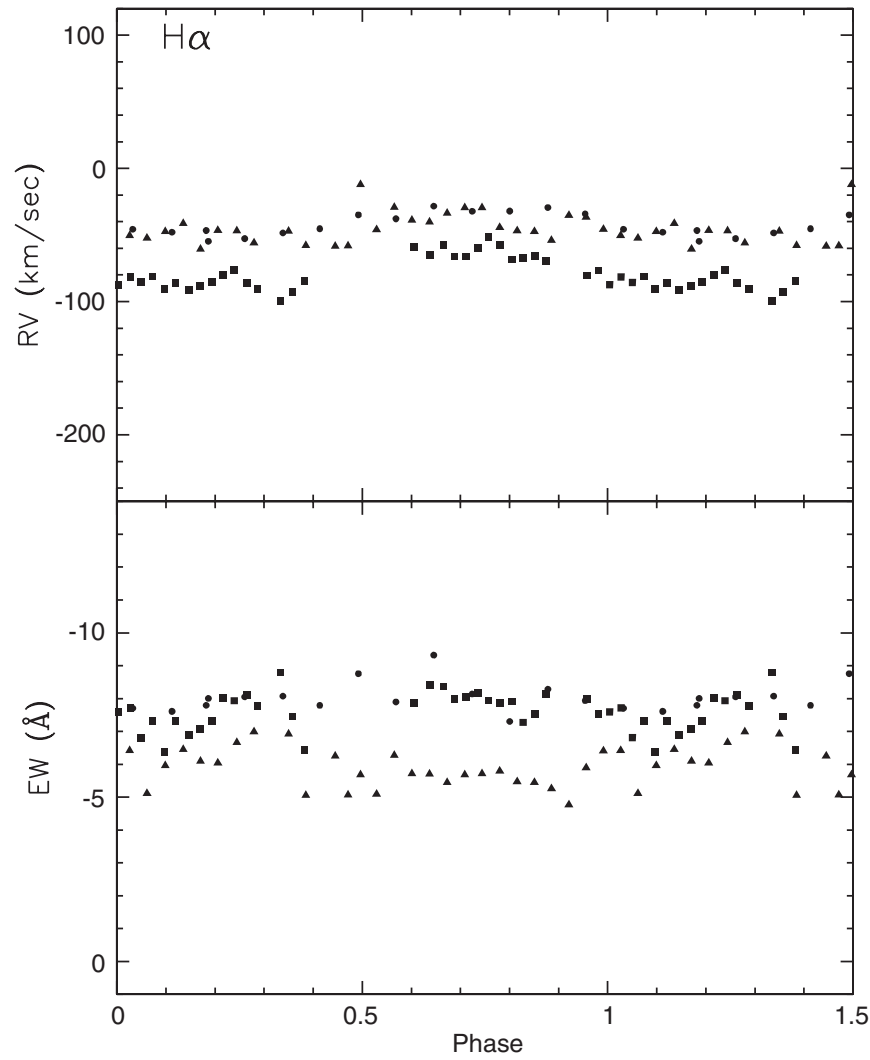

Figure 3. $\mathrm{RV}$ and $\mathrm{EW}$ of the $\mathrm{H} \alpha$ line. The symbols are the same as in Figure 2.
Table 3

Fits on the RVs of the Emission Lines for the Three Epochs of Observations

\begin{tabular}{lccc}
\hline \hline Line & $\gamma\left(\mathrm{km} \mathrm{s}^{-1}\right)$ & $K\left(\mathrm{~km} \mathrm{~s}^{-1}\right)$ & $\phi_{0}$ \\
\hline \multicolumn{4}{c}{$\mathrm{H} \alpha($ center $)$} \\
\hline 2005 Sep 9 & $37 \pm 1$ & $27 \pm 2$ & $0.020 \pm 0.011$ \\
2005 Oct 8 & $56 \pm 1$ & $21 \pm 4$ & $0.027 \pm 0.025$ \\
2005 Oct 11 & $73 \pm 1$ & $25 \pm 2$ & $0.015 \pm 0.014$ \\
\hline \multicolumn{4}{c}{ He I 5876 } \\
\hline 2005 Sep 9 & $-64 \pm 2$ & $54 \pm 11$ & $0.237 \pm 0.026$ \\
2005 Oct 8 & $-62 \pm 1$ & $66 \pm 8$ & $0.178 \pm 0.017$ \\
2005 Oct 11 & $-124 \pm 2$ & $57 \pm 7$ & $0.202 \pm 0.014$ \\
\hline \multicolumn{4}{c}{ He I 6678 } \\
\hline 2005 Sep 9 & $-29 \pm 1$ & $58 \pm 7$ & $0.169 \pm 0.017$ \\
2005 Oct 8 & $-51 \pm 1$ & $78 \pm 7$ & $0.173 \pm 0.012$ \\
2005 Oct 11 & $-73 \pm 2$ & $61 \pm 7$ & $0.159 \pm 0.015$
\end{tabular}

on different nights (Table 3). Such changes are often seen in NL CVs (especially in SW Sex stars), and may be due to the varying wind contributions to the line profiles.

The low inclination angle of V592 Cas allows visibility of all emission sites (accretion disk, hotspot, WD, and irradiated donor star) at all phases. For each spectral feature we measured, the $K$ velocities are similar (within error) at all epochs, as is the phasing of the orbital modulation; this allows grouping data of all nights to derive properties of the system. Note that there is no detectable orbital modulation of the $\mathrm{H} \alpha$ emission line (Figure 3). This comes as a surprise since the RV of the Balmer emission lines $(\mathrm{H} \beta$ and $\mathrm{H} \gamma)$ in V592 Cas show strong sinusoidal 


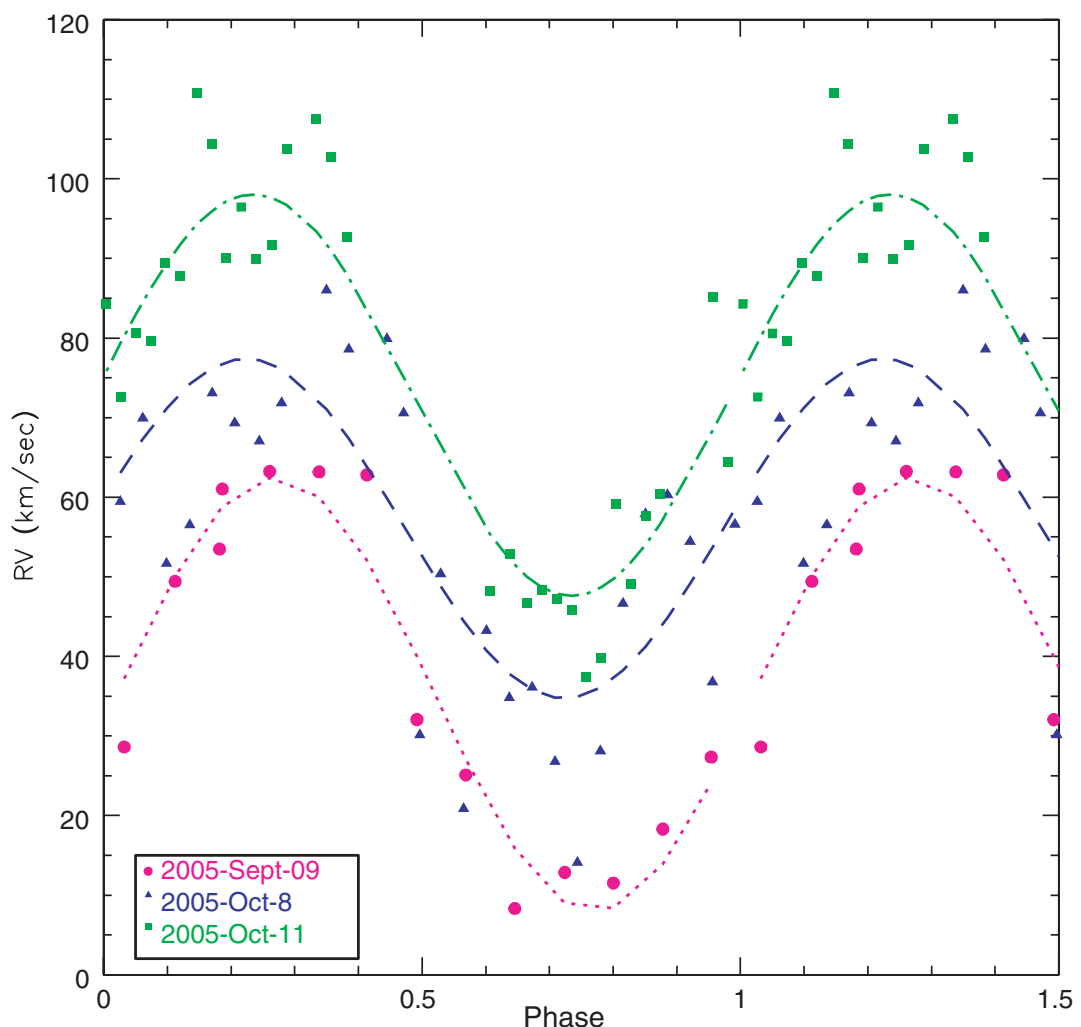

Figure 4. RV and fit of the central peak of $\mathrm{H} \alpha$ for the three epochs of observations; the relevant measurements appear in Table 4. Although all fits are in phase (indicating common origin of the central emission), the $\gamma$ and $K$ velocity vary. This demonstrates the presence of a variable component resulting in a variable central peak of $\mathrm{H} \alpha$ at different epochs.
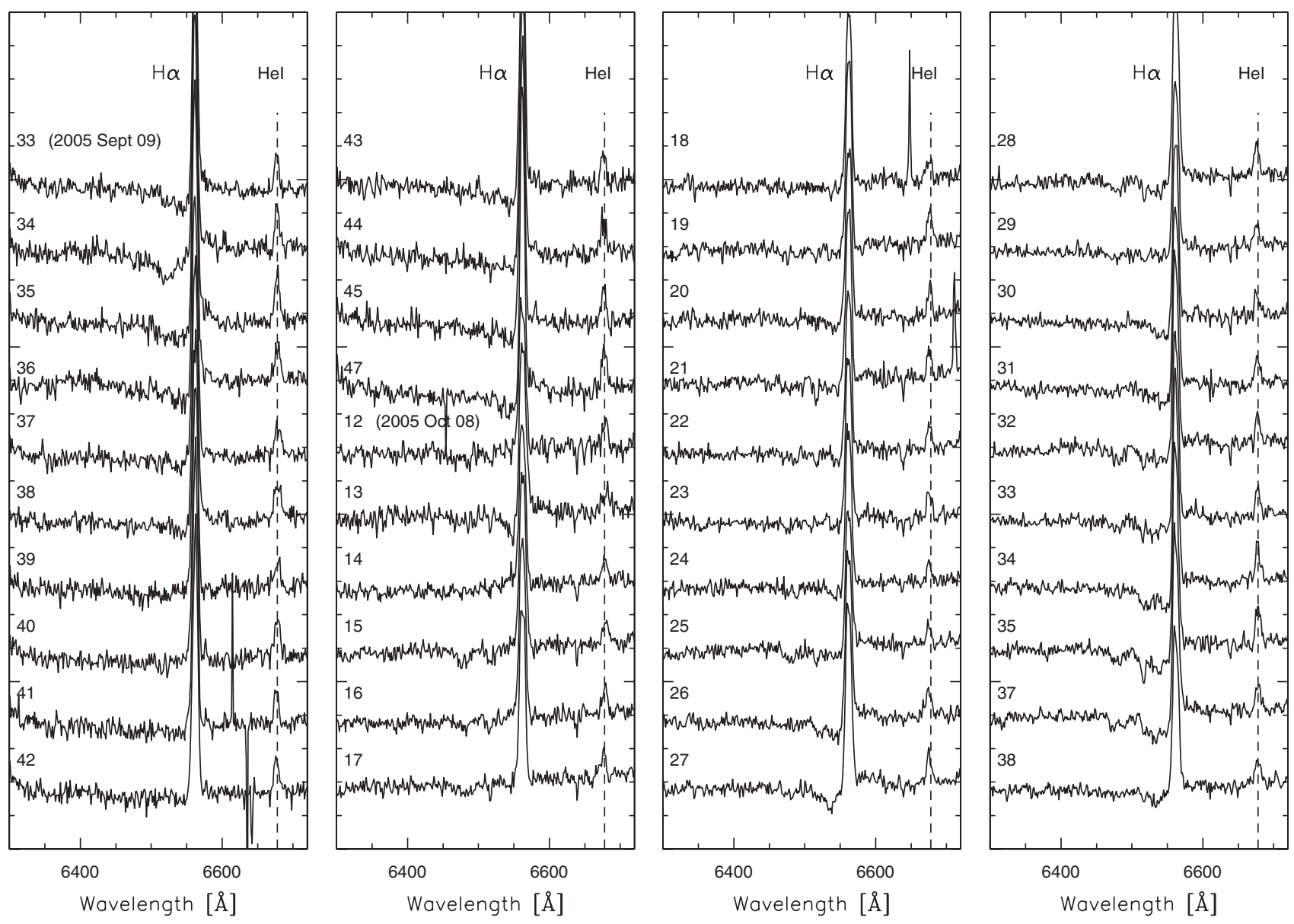

Figure 5. Nested spectra around the $\mathrm{H} \alpha$ line for all nights of our observations, demonstrating the time evolution of the H $\alpha$ P Cygni profiles. The last spectrum from each sequence is omitted because of its low $\mathrm{S} / \mathrm{N}$. 


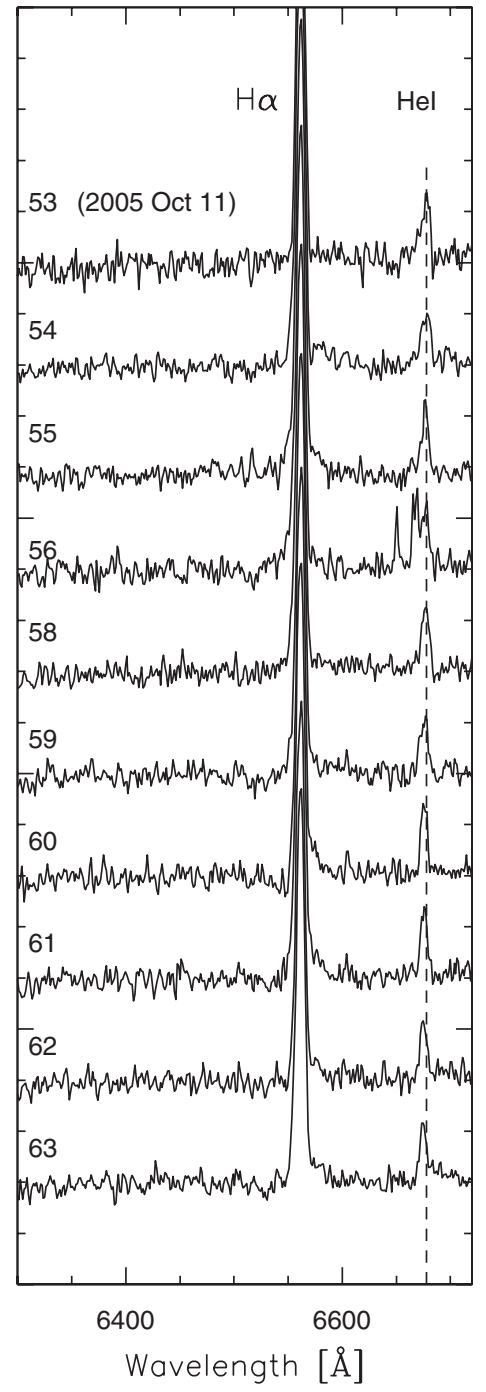

Wavelength $[\AA]$

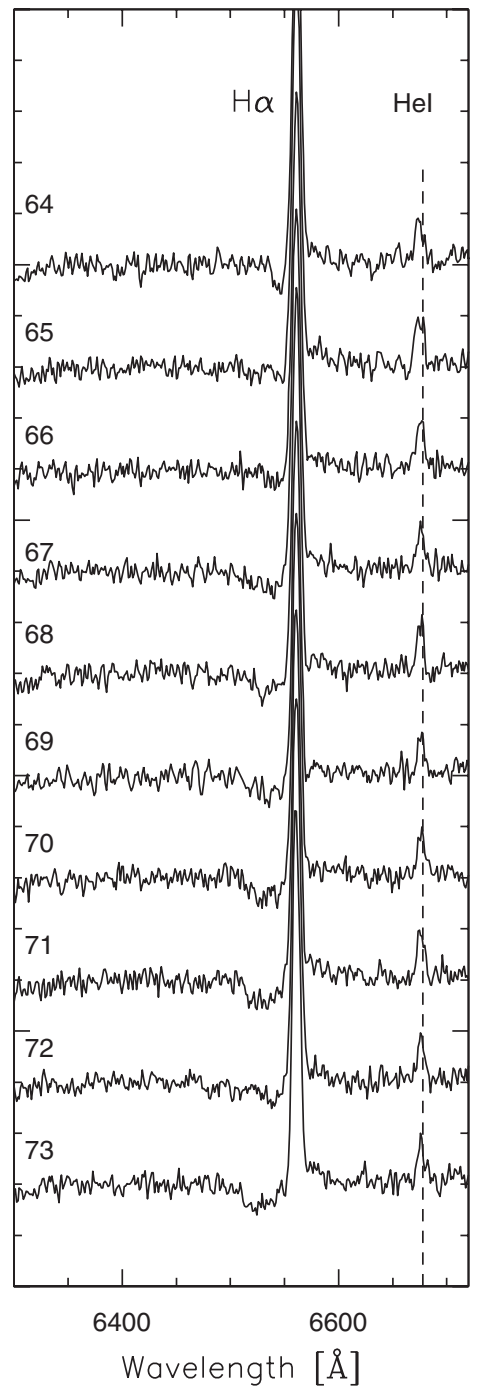

Figure 5. (Continued)

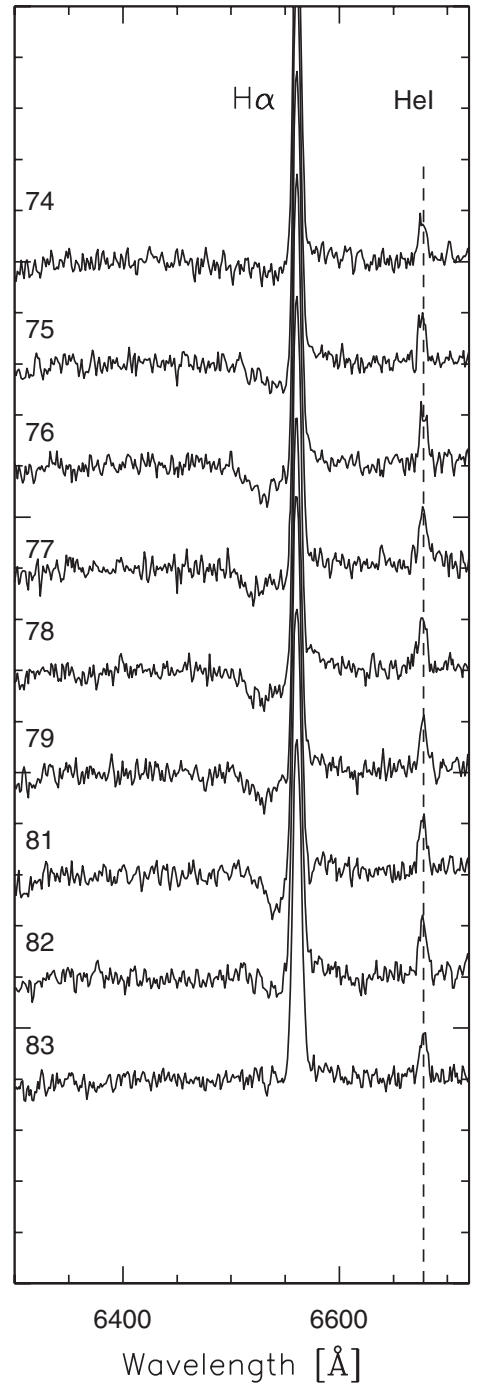

modulation with velocities reaching $K \sim 50 \mathrm{~km} \mathrm{~s}^{-1}$ (W03). In Figure 3, it appears that the systemic velocity of the $\mathrm{H} \alpha$ emission line changed by $\sim 40 \mathrm{~km} \mathrm{~s}^{-1}$ between nights. Based on the stability of the RV of telluric features, we think this detection is real. Because the winds in CVs are often highly variable, it is very likely that the apparent changing gamma is due to a varying wind contribution that affects the shape (and enhances the apparent gamma velocity) of the line profile from night-to-night. Focusing on the top 30\% of the $\mathrm{H} \alpha$ line (Figure 4 ) reveals an s-wave shifted by $\sim 0.1$ phase units with respect to the He I lines, with a variable systemic velocity for each of the nights of our observations (see Table 3). A phase shift between the RVs of the Balmer and He I lines is a characteristic commonly present in SW Sex type nova-like CVs (e.g., V442 Oph; Hoard et al. 2000), making V592 Cas a potential member of that class of CVs (although both T98 and W03 argue against this possibility and we acknowledge the difficulty of confirming a low-inclination $\mathrm{CV}$ a member of a group whose observational characteristics are most apparent in higher inclination CVs). Interestingly, W03 demonstrate that their $\mathrm{H} \beta$, He I 4471, and He II 4686 also show significant levels of variability near the line centers by about $\sim 2000 \mathrm{~km} \mathrm{~s}^{-1}$.

Figure 5 shows the $\mathrm{H} \alpha$ region in nested plots as a function of time for the three nights of our observations. The typical spacing of the WIYN spectra in Figure 5 is $700 \mathrm{~s}$, while the typical spacing of the $2.1 \mathrm{~m}$ red spectra is $240 \mathrm{~s}$. The spectral number is our internal numbering but is provided to denote the time order. In these nested spectra we see that the blue absorption wing is not always present in $\mathrm{H} \alpha$, consistent with the episodic character of the wind as seen in the optical (Kafka et al. 2003; Kafka \& Honeycutt 2004). When present, the profile of the blue absorption wind changes, being sometimes narrow, sometimes broad, and occasionally having two components (for example, see spectra 25 and 33 in Figure 5). The He I 6678 emission line does not show P Cygni, consistent with a disk origin of this line as deduced in Q Cyg (Kafka et al. 2003). No periodic or large amplitude EW variations are observed in any of the emission lines (Figures 2 and 3); this is expected due to the low inclination of the system. An attempt to fold the RVs and/or EW of the lines to any of the system superhump periods lead to null results.

\subsection{An Orbitally Modulated Outflow?}

Figure 1 shows a P Cygni profile in the blue wing of the $\mathrm{H} \alpha$ line; this is also seen in several of our individual spectra in Figure 5. Likewise, the He I $5876 \AA$ A emission line is occasionally accompanied by blueshifted absorption. Since the He I $6678 \AA$ 
Table 4

$\mathrm{H} \alpha$ Radial Velocity Measurements

\begin{tabular}{|c|c|c|c|}
\hline Spectrum ID ${ }^{\mathrm{a}}$ & HJD-2,453,622 & Phase & $\begin{array}{c}\mathrm{RV} \\
\left(\mathrm{km} \mathrm{s}^{-1}\right)\end{array}$ \\
\hline 33 & 0.88 & 0.112 & 49.4 \\
\hline 34 & 0.89 & 0.186 & 61.0 \\
\hline 35 & 0.90 & 0.261 & 63.2 \\
\hline 36 & 0.91 & 0.338 & 63.2 \\
\hline 37 & 0.92 & 0.413 & 62.8 \\
\hline 38 & 0.93 & 0.492 & 32.0 \\
\hline 39 & 0.94 & 0.569 & 25.1 \\
\hline 40 & 0.95 & 0.646 & 8.3 \\
\hline 41 & 0.96 & 0.724 & 12.8 \\
\hline 42 & 0.96 & 0.800 & 11.5 \\
\hline 43 & 0.97 & 0.878 & 18.3 \\
\hline 44 & 0.98 & 0.954 & 27.3 \\
\hline 45 & 0.99 & 0.032 & 28.6 \\
\hline 47 & 1.01 & 0.182 & 53.5 \\
\hline 12 & 29.81 & 0.471 & 70.6 \\
\hline 13 & 29.81 & 0.497 & 30.2 \\
\hline 14 & 29.81 & 0.529 & 50.4 \\
\hline 15 & 29.82 & 0.565 & 20.9 \\
\hline 16 & 29.82 & 0.601 & 43.2 \\
\hline 17 & 29.83 & 0.637 & 34.8 \\
\hline 18 & 29.83 & 0.673 & 36.1 \\
\hline 19 & 29.83 & 0.709 & 26.8 \\
\hline 20 & 29.84 & 0.744 & 14.1 \\
\hline 21 & 29.84 & 0.780 & 28.1 \\
\hline 22 & 29.85 & 0.815 & 46.6 \\
\hline 23 & 29.85 & 0.851 & 57.9 \\
\hline 24 & 29.85 & 0.886 & 60.3 \\
\hline 25 & 29.86 & 0.921 & 54.4 \\
\hline 26 & 29.86 & 0.956 & 36.8 \\
\hline 27 & 29.87 & 0.991 & 56.6 \\
\hline 28 & 29.87 & 0.026 & 59.4 \\
\hline 29 & 29.87 & 0.061 & 69.9 \\
\hline 30 & 29.88 & 0.099 & 51.7 \\
\hline 31 & 29.88 & 0.135 & 56.5 \\
\hline 32 & 29.89 & 0.171 & 73.1 \\
\hline 33 & 29.89 & 0.206 & 69.3 \\
\hline 34 & 29.90 & 0.244 & 67.1 \\
\hline 35 & 29.90 & 0.279 & 71.8 \\
\hline 37 & 29.91 & 0.350 & 86.0 \\
\hline 38 & 29.92 & 0.385 & 78.6 \\
\hline 53 & 32.81 & 0.606 & 48.2 \\
\hline 54 & 32.82 & 0.639 & 52.9 \\
\hline 55 & 32.82 & 0.665 & 46.7 \\
\hline 56 & 32.82 & 0.688 & 48.4 \\
\hline 57 & 32.83 & 0.712 & 47.2 \\
\hline 58 & 32.83 & 0.735 & 45.8 \\
\hline 59 & 32.83 & 0.758 & 37.4 \\
\hline 60 & 32.83 & 0.781 & 39.8 \\
\hline 61 & 32.84 & 0.805 & 59.2 \\
\hline 62 & 32.84 & 0.828 & 49.1 \\
\hline 63 & 32.84 & 0.851 & 57.6 \\
\hline 64 & 32.85 & 0.874 & 60.3 \\
\hline 65 & 32.85 & 0.957 & 85.2 \\
\hline 66 & 32.86 & 0.981 & 64.4 \\
\hline 67 & 32.86 & 0.004 & 84.3 \\
\hline 68 & 32.86 & 0.027 & 72.6 \\
\hline 69 & 32.87 & 0.050 & 80.6 \\
\hline 70 & 32.87 & 0.073 & 79.6 \\
\hline 71 & 32.87 & 0.097 & 89.4 \\
\hline 72 & 32.87 & 0.120 & 87.8 \\
\hline 73 & 32.88 & 0.146 & 110.7 \\
\hline 74 & 32.88 & 0.170 & 104.4 \\
\hline 75 & 32.88 & 0.193 & 90.1 \\
\hline 76 & 32.88 & 0.216 & 96.5 \\
\hline 77 & 32.89 & 0.239 & 89.9 \\
\hline
\end{tabular}

Table 4

(Continued)

\begin{tabular}{lccr}
\hline \hline Spectrum ID $^{\mathrm{a}}$ & HJD-2,453,622 & Phase & $\begin{array}{c}\mathrm{RV} \\
\left(\mathrm{km} \mathrm{s}^{-1}\right)\end{array}$ \\
\hline 78 & 32.89 & 0.264 & 91.7 \\
79 & 32.89 & 0.287 & 103.8 \\
81 & 32.90 & 0.334 & 107.5 \\
82 & 32.90 & 0.357 & 102.8 \\
83 & 32.90 & 0.382 & 92.8 \\
\hline
\end{tabular}

Note.

a The spectrum ID is the same as the number presented in Figure 5.

line does not display similar absorption, we can exclude the accretion disk and the WD as the origin of the absorption in He I. The character of the wind in the He I $5876 \AA$ A seems to be different than in $\mathrm{H} \alpha$; however, the presence of C IV $5801 \AA$ and $5812 \AA$ emission does not allow measuring wind velocity from the absorption blueward of $\mathrm{He}$ I $5876 \AA$. In $\mathrm{H} \alpha$ the wind shows multiple episodes reaching velocities of $\sim 5000 \mathrm{~km} \mathrm{~s}^{-1}$. Because the blue absorption profile is both complicated and changeable, we simply integrated between -2500 and $1500 \mathrm{~km} \mathrm{~s}^{-1}$ to obtain an EW for the absorption; this is the same velocity range used in W03. Figure 6 shows the results for our three nights of data. The EW behavior is episodic, with relatively long intervals of no measurable wind punctuated by a single active interval in each data set. These activity episodes have typical durations of 3040 minutes, similar to the $\mathrm{H} \alpha$ wind event in Q Cyg (Kafka et al. 2003). In the W03 study, the blue absorption at $\mathrm{H} \beta$ was found to be modulated with a period similar to the orbital period, with the difference tentatively attributed to wobbling of the accretion disk. In the FUSE far-UV spectra of V592 Cas, Prinja et al. (2004) found that the C III $1176 \AA$ line is strongly modulated with orbital phase, indicating a departure from axisymmetry in the outflow. They discuss possible explanations (e.g., a magnetic $\mathrm{WD}$, overflowing gas in the disk, spiral density waves in the disk) without favoring any of them.

In the UV study of Prinja et al. (2004), the C III $1176 \AA$ line of V592 Cas displays its maximum EW for half of the orbit. To investigate whether the outflow in the optical is also phase-modulated, we binned and median combined our spectra in increments of 0.1 phase units. We then looked for patterns with phase or time in the presence, variations, and strength of the absorption wind signature. Figure 6 shows that wind events during the three nights occurred near orbital phases $0.8,0.9$, and 0.1. Our individual data sets each span less than a complete orbital cycle, but the reoccurence of this behavior in three data sets obtained many orbital cycles apart suggests that it is a persistent orbital phase dependence. Our spectra suggest that a similar phase-dependence is present in He I $5876 \AA$ A; however, the phase interval in which the outflow is present changes at different epochs. For example, all of the spectra from 2005 October 11 show the presence of an outflow associated with the He I $5876 \AA$ line. This agrees with the behavior of the old nova Q Cyg, in which P Cygni profiles are always present in the He I triplets, whereas they appear to be episodic in $\mathrm{H} \alpha$ (Kafka et al. 2003). This also implies that the source of the outflow seen in these two lines is different. A possibility is that disk precession and/or spiral waves modulate the outflow axis (as suggested by W03); this would lead to periodic variations of the outflow on one of the superhump periods, which is not observed in our data (nor is demonstrated in the W03 data). The only sites 

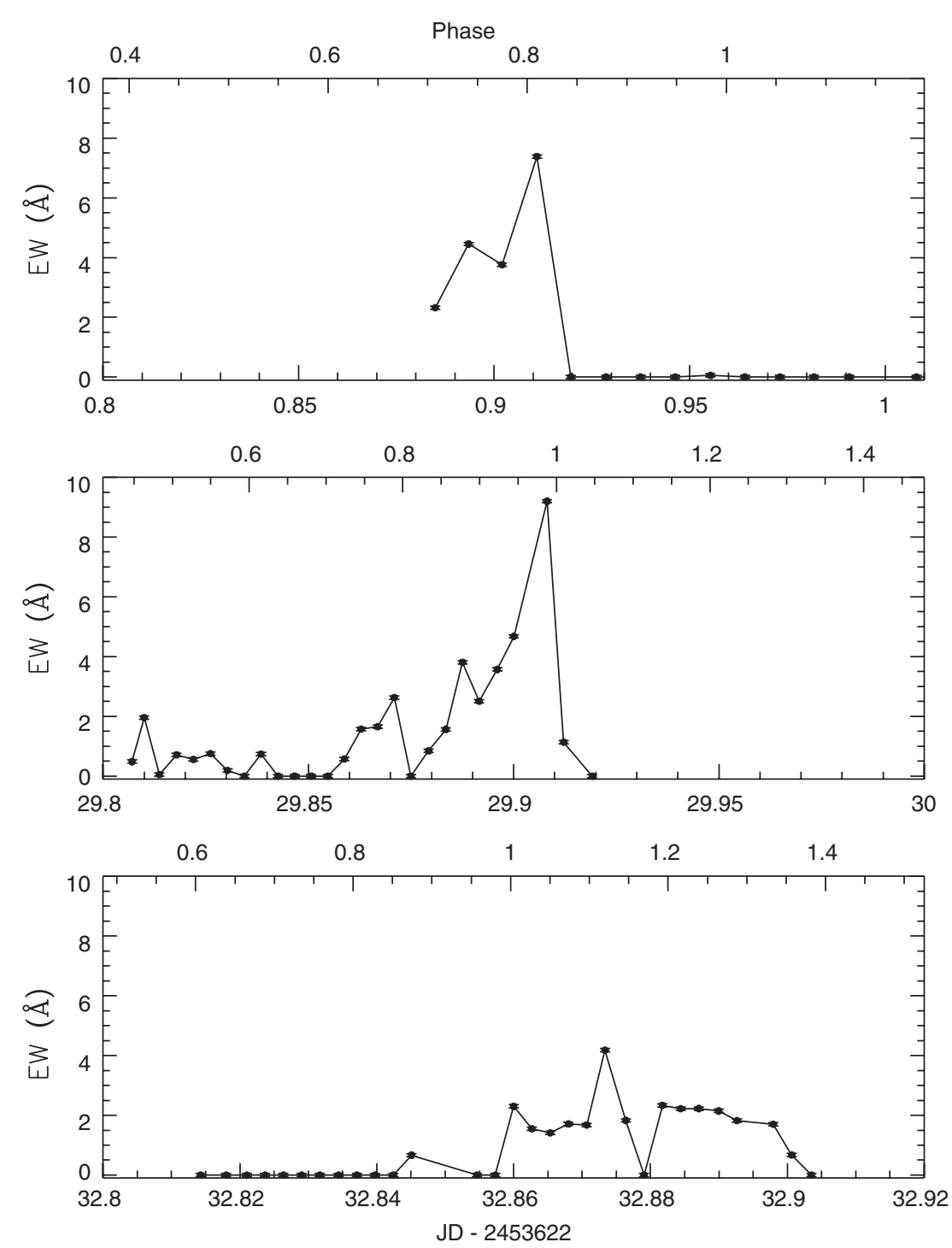

Figure 6. EWs of the blueshifted absorption (P Cygni) in the $\mathrm{H} \alpha$ line for the three nights of our spectroscopic observations. The top axis in each panel corresponds to phase, whereas the bottom panel is time (JD-2453622). See the text for discussion.

in the system that can produce an outflow in which the wind propagation axis is tilted with respect to the orbital plane (thus "breaking" the wind axisymmetry) are the bright spots created by the initial impact of the stream on the accretion disk rim and/or at a secondary site within the disk in the case of an overflowing stream (as suggested for the SW Sex stars; see Hoard et al. 2003 and references therein). Both sites are energetically capable of producing an outflow with RVs visible only at certain orbital phases along the line-of-sight of the observer. Because we are unable to reliably associate orbital phase with viewing angle, we cannot discriminate between these two locations using our data.

\subsection{Correlations with Photometry?}

There is very little information on whether wind events in CVs are correlated with photometric variations, which could shed light on the origin of the wind. Kafka et al. (2003) suggested that enhanced wind events in Q Cyg might be correlated with stunted outbursts seen in $V$ band. Flickering timescales in nova-like $\mathrm{CVs}$ such as V592 Cas overlap (at the slow end) with timescales of individual wind events. The origin of the flickering appears to be the inner disk in some CVs and the stream-impact bright spot on the disk in others (Welsh et al. 1997; Bruch 2000), locations that have also been suggested for the origin of the wind.

During our 2005 October 8 spectroscopic observations of V592 Cas, we obtained simultaneous photometry covering $\sim 1.8 \mathrm{hr}$ using the WIYN $0.9 \mathrm{~m}$ telescope. The average brightness of V592 Cas is $\langle V\rangle=12.55$ with $\sim 0.2$ mag quasiperiodic variations on timescales of minutes, similar to the behavior presented in Kato \& Starkey (2002). Periodogram analysis of our data and the white light AAVSO light curve of V592 Cas did not recover the 21-minute period reported in Kato \& Starkey (2002) nor any other periodic signal. The left panels of Figure 7 show the individual spectra taken during our photometric observations. Strong P Cygni profiles are present in all but one of the spectra (No. 2), with some spectra (No. 5, No. 9) showing multiple P Cygni dips at different velocity offsets, indicative of multiple successive outflow episodes. The spectra are numbered in sequence and these numbers are plotted at the times of observation in the top right panel of Figure 7, which also shows the $V$-band light curve. We measured the maximum velocity of the absorption profiles in $\mathrm{H} \alpha$ using the IRAF 

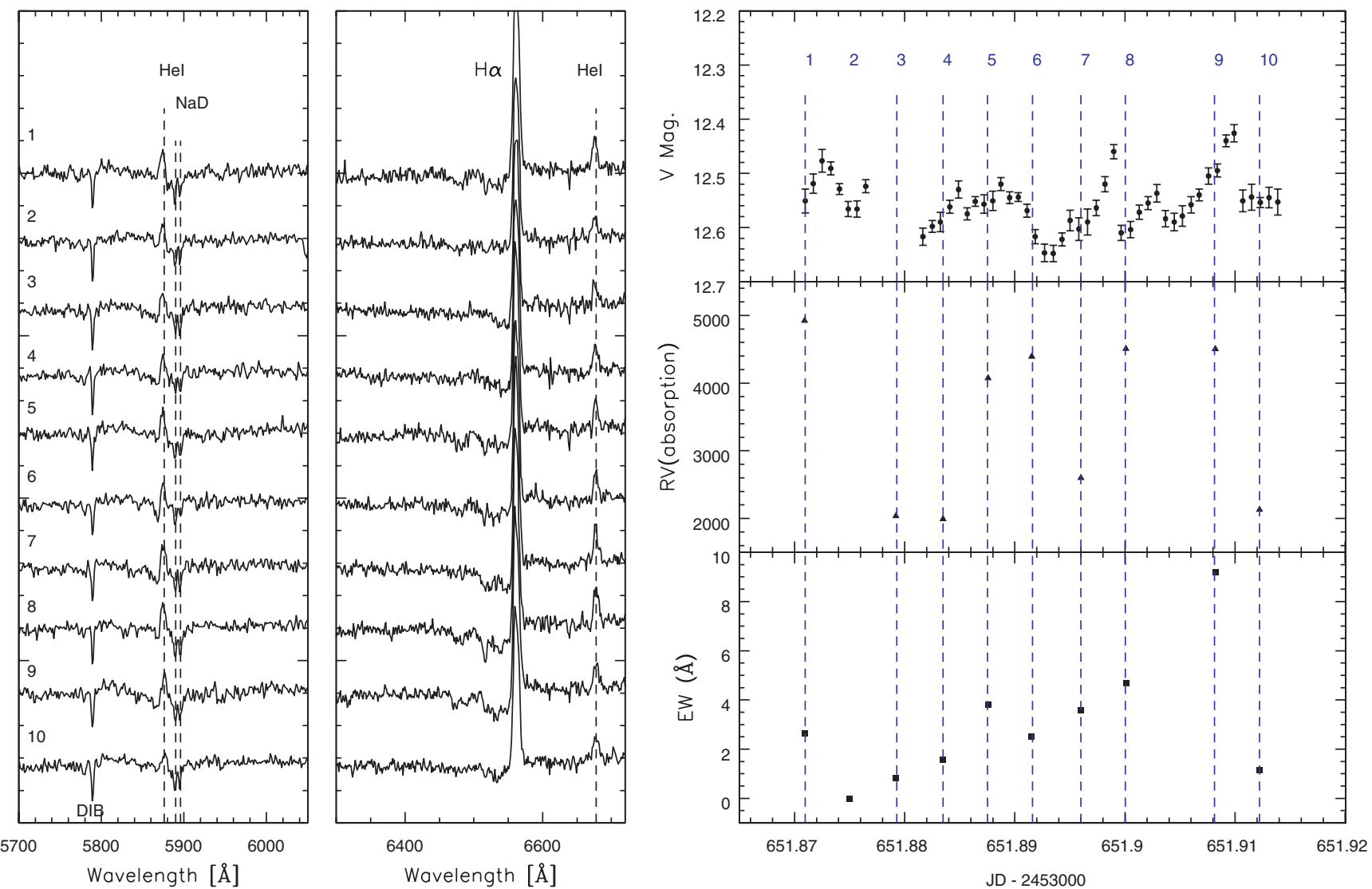

Figure 7. Left and middle: spectra of V592 Cas at the time of the photometric observations. The spectra are corrected for telluric features, normalized to unity, and offset by a constant for clarity. Right, top: $V$-band light curve of V592 Cas. The number labels correspond to the times of observation of the correspondingly numbered spectra in the left panels. Right, middle: maximum RV of the blueshifted absorption (P Cygni) profiles at H $\alpha$. Right, bottom: total EWs of the blueshifted absorption at $\mathrm{H} \alpha$. The vertical dashed lines in the right panels show the times of observation of the spectra that display blueshifted absorption features at the H $\alpha$ and $\mathrm{He}$ I $5876 \AA$ lines.

task splot and cursor positioning ${ }^{6}$. There is no consistently convincing correlation between the maximum velocity of the absorption and the brightness of the $\mathrm{CV}$ (the middle right panel of Figure 7). We also measured the EW of the wind absorption in the $\mathrm{H} \alpha$ line using IRAF/splot (the bottom right panel of Figure 7). Again, there is no convincing correlation between the absorption EW and the brightness of the system. Taken together, these results imply that the characteristics of the wind (as expressed in $\mathrm{H} \alpha$ ) are not related to the source of the $V$-band photometric modulations in V592 Cas.

\section{SUMMARY}

In this paper we present an optical spectroscopic study of V592 Cas, aimed at exploring the presence of an outflow in the optical spectra. In the red, the system has all the characteristics of a low inclination disk $\mathrm{CV}$, with all the $\mathrm{He} \mathrm{I}$ and $\mathrm{H} \alpha$ lines in emission. P Cygni profiles, indicative of an outflowing wind, are present in the blue wing of the $\mathrm{H} \alpha$ and, more weakly, He I $5876 \AA$ emission lines in many of our individual spectra; however, no such absorption is present in the He I $6678 \AA$ line in any of our spectra. Apparent variations in the systemic velocity of the emission line RV curves likely stem from the contamination of the emission line profiles by a P Cygni

\footnotetext{
6 With this method, the error in determining the maximum velocities in the spectra is of the order of $200 \mathrm{~km} \mathrm{~s}^{-1}$, an order of magnitude smaller than the measured outflow velocities. Thus, the fact that these measurements were performed by visual inspection is of no consequence to the results.
}

component (i.e., wind) of different strength on different nights, rather than an actual change in the overall systemic velocity or accretion flow in the CV. The wind in the $\mathrm{H} \alpha$ line is episodic, with events having duration significantly less than the orbital period of the CV. The observed maximum outflow velocities approach $\sim 5000 \mathrm{~km} \mathrm{~s}^{-1}$. Some of the P Cygni profiles show multiple dips at different velocities, indicative of successive outflow events. There is some indication that the $\mathrm{H} \alpha$ outflow is present only in a specific orbital phase range $(\sim 0.8-0.1)$, in agreement with relevant studies in the UV suggesting that the wind in V592 Cas is phase modulated. If the phase dependence of the P Cygni profiles is confirmed, then a possible origin for this component of the outflow is a bright spot on the edge and/or interior of the accretion disk. An observed phase shift between the RVs of $\mathrm{He}$ I and the central $\mathrm{H} \alpha$ line is similar to the characteristic behavior of the SW Sextantis stars (Hoard et al. 2003 and references therein). Definitive classification of V592 Cas as an SW Sex star is beyond the scope of our current data. However, we note that this would be consistent with a possible scenario for the origin of the outflow in V592 Cas in a secondary bright spot in the disk interior at the impact site of an overflowing accretion stream. Simultaneous optical photometry during one night of our spectroscopic observations does not show any convincing correlation between the observed photometric variations and the presence or strength of the wind in V592 Cas.

One of the aims of our work is to examine if the observed outflow can be accounted for angular momentum loss in this 
system. In the literature the relevant models calculating the mass and angular momentum loss rate due to outflows from CVs are applicable for species such as the UV Si v and C IV and take into account their ionization fraction, line shape, and observed velocity in the magnetohydrodynamic computations. Those calculations use the P Cygni absorption profiles in UV resonance lines, the mass of the WD (usually assumed to be $\left.M_{\mathrm{WD}}=0.6 M_{\mathrm{sun}}\right)$ a certain mass-radius relationship for the donor star and two free parameters, namely the $M$ and the inclination $i$ of the system, demonstrating the effects of different combinations of the latter two on the mass loss rate and angular momentum loss due to the wind (e.g., Proga \& Kallman 2002). Of the key assumptions of the calculations is that the wind is bipolar and axisymmetric, originating on the inner or outer part of the accretion disk. As suggested in this paper (and in Prinja et al. 2004), the wind in V592 Cas is phase modulated, indicating a source with a varying visibility. Thus the existing theoretical calculations for angular momentum loss in CVs cannot be applied in V592 Cas. Therefore in V592 Cas, we cannot derive wind properties (such as the mass loss rate) and assess if those episodes can be responsible for angular momentum loss from the system. Since V592 Cas is not the only CV with phasedependent outflows in the UV resonance lines (Prinja et al. 2004), there is a pressing need for a model that takes into account a nonaxisymmetric wind in order to examine if such a wind can be responsible for mass and angular momentum loss in these systems driving their evolution.

We thank our anonymous referee for her/his thorough review of the manuscript.

Facilities: KPNO 2.1 m, WIYN 3.5 m, WIYN 0.9 m.

\section{REFERENCES}

Bruch, A. 2000, A\&A, 359, 998

Cannizzo, J. K., \& Pudritz, R. E. 1988, ApJ, 327, 840

Downes, R., Hoard, D. W., Szkody, P., \& Wachter, S. 1995, AJ, 110, 1824

Greenstein, J. L., Sargent, A. I., \& Haug, U. 1970, A\&A, 7, 1

Henden, A. A., \& Honeycutt, R. K. 1995, PASP, 107, 324

Hoard, D. W., Szkody, P., Froning, C. S., Long, K. S., \& Knigge, C. 2003, AJ, 126,2473

Hoard, D. W., Thorstensen, J. R., \& Szkody, P. 2000, ApJ, 537, 936

Hollis, J. M., Oliversen, R. J., Wagner, R. M., \& Feibelman, W. A. 1992, ApJ, 393, 217

Honeycutt, R. K. 1992, PASP, 104, 435

Honeycutt, R. K., Schlegel, E. M., \& Kaitchuck, R. H. 1986, ApJ, 302, 388

Huber, M. E., Howell, S. B., Ciardi, D. R., \& Fried, R. 1998, PASP, 110, 784

Kafka, S., Anderson, R., \& Honeycutt, R. K. 2008, AJ, 135, 1649

Kafka, S., \& Honeycutt, R. K. 2004, AJ, 128, 2420

Kafka, S., Tappert, C., Honeycutt, R. K., \& Bianchini, A. 2003, AJ, 126, 1472

Kato, T., \& Starkey, D. R. 2002, Inf. Bull. Variable Stars, 5358, 1

Marsh, T. R., \& Horne, K. 1990, ApJ, 349, 593

Prinja, R. K., Knigge, C., Witherick, D. K., Long, K. S., \& Brammer, G. 2004, MNRAS, 355, 137

Proga, D. 1999, MNRAS, 304, 938

Proga, D., \& Kallman, T. R. 2002, ApJ, 565, 455

Ringwald, F. A., \& Naylor, T. 1998, AJ, 115, 286

Spruit, H. C., \& Ritter, H. 1983, A\&A, 124, 267

Taam, R. E., \& Spruit, H. C. 2001, ApJ, 561, 329

Taylor, C. J., et al. 1998, PASP, 110, 1148, T98

Wallace, L., \& Livingston, W. 2003, An Atlas of the Solar Spectrum in the Infrared from 1850 to $9000 \mathrm{~cm}^{-1}$ (1.1 to 5.4 micrometer), NSO Technical Report (revised ed.; Tucson, AZ: National Solar Observatory, National Optical Astronomy Observatory)

Welsh, W. F., Wood, J. H., \& Horne, K. 1997, IAU Colloq. 163: Accretion Phenomena and Related Outflows, 121, 331

Witherick, D. K., Prinja, R. K., Howell, S. B., \& Wagner, R. M. 2003, MNRAS, 346,861 , W03 